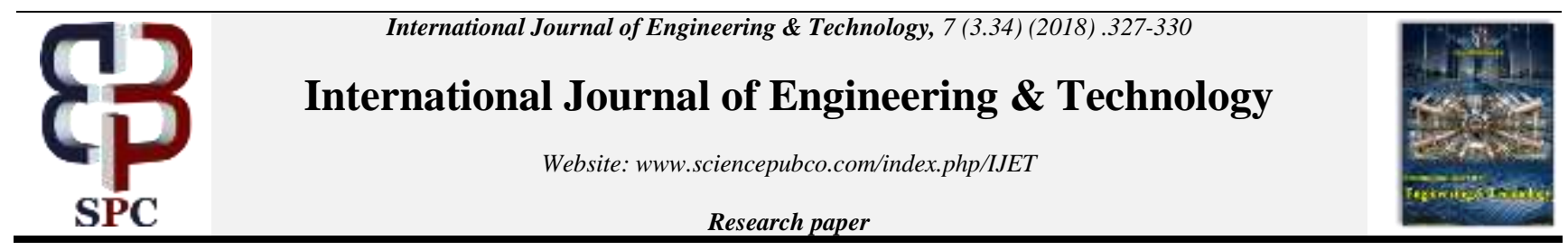

\title{
Image Denoising in Wavelet Domain with Filtering and Thresholding
}

\author{
${ }^{1} \mathrm{~K} . S u^{\mathrm{ma}}$ thi, ${ }^{2}$ Ch.Hima Bindu \\ ${ }^{I}$ ECE Department, QISCET, Ongole.prakasam,A.P.,India \\ ${ }^{2}$ IEEE,ACM Member QISCET, Ongole. Prakasam, A.P., India \\ *Corresponding author E-mail: ${ }^{1}$ sumapannem@gmail.com, ${ }^{2}$ gghb.muvvala@gmail.com
}

\begin{abstract}
In this paper, the proposed method is implemented for removal of salt \& pepper and Gaussian noise of black \& white $\&$ color images to acquire the quality output. In this work initially wavelet coefficients are extracted for noisy images. Later apply denoise filtering technique on the high transform sub bands of noisy images (either color/ B \& W) using new laplacian filters with 4 directions. Finally threshold of an image is generated to extract denoisy coefficients. At last inverse of above subband coefficients can give denoise image for further processing. The proposed method is verified against various B \& W/color images and it gives a better PSNR (Peak Signal to Noise Ratio) \& MI (Mutual Information). These values are compared with different noise densities and analyzed visually.
\end{abstract}

Keywords: Enhancement,Discrete wavelet TransformationDenoisingfilters, Threshold.Virtual reality.

\section{Introduction}

Image enhancement is a method of improving the quality of an image. The main objective of image enhancement is to change the characteristics of an image to get more suitable for observer and better input for other automated image processing techniques. During this process, one or more characteristics of the image are modified. Moreover the human visual system and the observer's experience, will introduce a great deal of subjectivity into the choice of image enhancement methods [2,3].

The enhancement methods are classified in to the following two categories:

\section{Spatial Domain Methods}

\section{Transform Domain Methods}

In spatial domain method [4], pixel values are directly calculated to get better improvement of an image enhancement. The images are first transformed into transform domain and later transformed coefficients are enhanced to obtain desired output in transform domain. In both methods we achieve brightness, contrast, or gray levels distribution of an image with enhancement techniques [5]. To understand and analyze the image in various fields like medical imaging, analysis of images from satellites and IR etc [2, 3].

The images that are collected from various image sensors by noisy images because imperfect devices and problem with data acquisition process introducing interference. So the data can degrade in terms of quality. Moreover, transmission errors and compression introduces noise [1]. Thus, it is necessary to denoising the data, before images data is analyzed. Denoising technique is useful to get efficient output from corrupted data. Still remains a challenge for researchers Image denoising is a big task to improve the quality of an image because it introduces blurring of the images [6].
The rest of the paper is managed in 6 sections. The section II addressed about image denoising technique and literature survey, the section III addressed about Discrete Wavelet Transformation, in section IV discussion on proposed work, in section $\mathrm{V}$ discussion on experimental results. Finally the paper is concluded with resultant future scope in section VI.

\section{Image Denoisisng}

Image denoising is a process of mitigating noise from an noisy image. Based on type of noise affected by the devices or processing methods. The important function of image denoising is the removal of noise while preserving edge details. A wide variety of non linear median type filter has been developed to get better quality of an image[4,8].

By using wavelet based denoising techniques improved the weakness of the spatial filters [4]. using FFT with LPF dennoising an image. With help of transform domain filters, design removal of noise is achieved[8]. When noise components are separated from a signal by adapting an cut off in transform domain.

For denoising, information about noise the basic step but practically this is not possible. The limitation of FFT in filtering is sparse representation of data. So to overcome this draw back by performing wavelet transformations since wavelet transformation has sparsity, multi resolution and multi scale nature. Moreover wavelet transformation reduces the mathematical calculations. Thresholding with [8] and multi wavelet transformations raise the performance in non orthogonal wavelet transformation. HMM models are efficient but more complex[13]. Better shrinkage of noise in an image by using wavelet transformation [7, 11]. Many of Existing algorithms like VMF and AVMF, the noisy pixels are replaced by its median values [7]. VMF was improved by using variable Threshold. AVMF detect the probability noisy pixel with threshold value and filter does not replace by pixel unlike the 
VMF filter [11].

\section{Discrete Wavelet Transform}

DWT is one of the most widely used transform method for image processing. It is used to overcome all the drawbacks that are occurred when using the basic image like Fourier transforms, Fourier series techniques etc, with the functionality of scaling \& wavelet concepts.

DWT uses wavelet filters for the transformation of an image. Some of the wavelet filters are like orthogonal filters, haar wavelet filter \& bi orthogonal filters.

Dwt uses wavelet decomposition principle, the decomposition can be done in multi levels called multi resolution domain. After applying DWT, the image is divided into 4 sub bands, Like LL (Low Low), LH (Low High), HL (High Low), HH (High High), when the image is passed through a series of high pass, low pass filters. Most of the original image is lies in LL sub band and the remaining sub bands contain the edges of the image. the applying of output by using the low pass filter in horizontal direction \& the high pass filter in vertical direction gives the LL sub band. The LL sub band contains more information and it is useful in the extraction process.

The two dimensional DWT $[4,15]$ of an image $f(x, y)$ is given as

$$
\begin{aligned}
& f(u, v)=\frac{1}{\sqrt{S T}} \sum_{s} \sum_{t} W_{\varphi}\left(y_{0,} s, t\right) \varphi_{y 0, s, t}(u, v)+ \\
& \frac{1}{\sqrt{S T}} \sum_{x=H, V, D} \sum_{y=y_{0}}^{\infty} \sum_{s} \sum_{t} W_{\varphi}^{x}(y, s, t) \varphi_{y, s, t}^{x}(u, v)
\end{aligned}
$$

Where the wavelet coefficients are

$$
\begin{aligned}
& W_{\varphi}\left(y_{0}, s, t\right)=\frac{1}{\sqrt{S T}} \sum_{u=0}^{S-1} \sum_{v=0}^{T-1} f(u, v) \varphi_{y_{0}, s, t}(u, v) \\
& W_{\varphi}^{x}(y, s, t)=\frac{1}{\sqrt{S T}} \sum_{u=0}^{S-1} \sum_{v=0}^{T-1} f(u, v) \varphi_{y, s, t}^{x}(u, v) \\
& x=\{H, V, D\}
\end{aligned}
$$

In two dimensions, a scaling function $\varphi(u, v)$ and three twodimensional wavelets $\psi^{H}(u, v), \psi^{V}(u, v),{ }_{\&} \psi^{D}(u, v)$ are required. Each is the product of two one dimensional functions. The wavelets are represented as

$$
\begin{aligned}
& \varphi(u, v)=\varphi(u) \varphi(v) \\
& \psi^{H}(u, v)=\psi(u) \varphi(v) \\
& \psi^{V}(u, v)=\varphi(u) \psi(v) \\
& \psi^{D}(u, v)=\psi(u) \psi(v)
\end{aligned}
$$

The decomposition of 1-D dwt is shown below as:

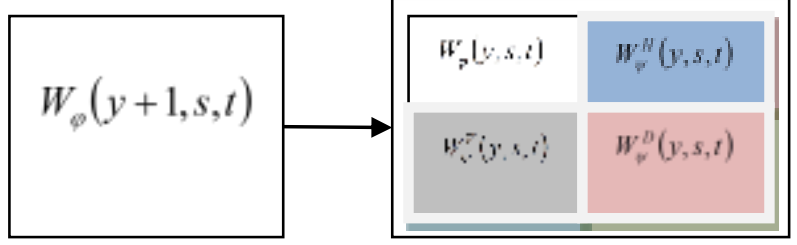

Figure 1:.Decomposition of 1-D DWT.

The DWT structure with labeled sub bands is shown below:

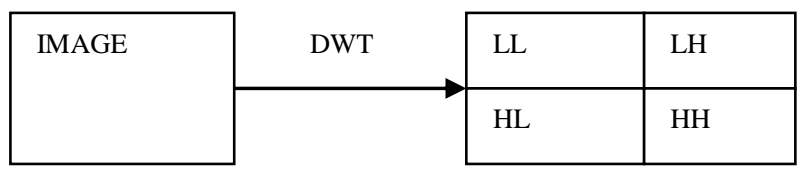

Figure 2.DWT structure with labeled sub bands.

\section{Proposed Method}

The quality of an image can be enhanced by using the following proposed method. The proposed method consists of six stages: Filtering and Thresholding. In first stage the noisy image is convolved with four laplacian operators. This filtered image can be threshold to obtain denoisy image efficiently. The flow of proposed method is shown in figure. 1 The procedure is as follows:

Step1: Consider the noisy image $\mathrm{I}_{\mathrm{N}}$ as input of the proposed method. The original image (I) is degraded by either salt and pepper or Gaussian noise to obtain $\mathrm{I}_{\mathrm{N}}$

Step2: Apply 1-level DWT on the noisy image to extract four sub bands of image.

$$
\begin{aligned}
& I_{N}=D W T\left(I_{N}\right) \\
& I_{N} \in\left(L L_{I_{N}}, L H_{I_{N}}, H L_{I_{N}} \& H H_{I_{N}}\right)
\end{aligned}
$$

Step3: The above high transform coefficients (HL, LH,HH) are convolved with four laplacian operators. We know that the high transformed sub bands bear the noise content. This can be

\begin{tabular}{|c|c|c|c|c|c|c|c|c|c|}
\hline 0 & 0 & 0 & 0 & -1 & 0 & 0 & -1 & 0 & 0 \\
\hline 0 & 0 & 0 & -1 & 0 & 0 & 0 & -1 & 0 & 0 \\
\hline 0 & 0 & 4 & 0 & 0 & 0 & 0 & 4 & 0 & 0 \\
\hline 0 & -1 & 0 & 0 & 0 & 0 & 0 & -1 & 0 & 0 \\
\hline \multirow[t]{3}{*}{-1} & 0 & 0 & 0 & 0 & 0 & 0 & -1 & 0 & 0 \\
\hline & & (a) & & & & & (b) & & \\
\hline & & & & & & & & & \\
\hline-1 & 0 & 0 & 0 & 0 & 0 & 0 & 0 & 0 & 0 \\
\hline 0 & -1 & 0 & 0 & 0 & 0 & 0 & 0 & 0 & 0 \\
\hline 0 & 0 & 4 & 0 & 0 & -1 & -1 & 4 & -1 & -1 \\
\hline 0 & 0 & 0 & -1 & 0 & 0 & 0 & 0 & 0 & 0 \\
\hline \multirow[t]{2}{*}{0} & 0 & 0 & 0 & -1 & 0 & 0 & 0 & 0 & 0 \\
\hline & & (C) & & & & & (d) & & \\
\hline
\end{tabular}
presented as:

$$
Z_{L H}(i, j)=\left(L H_{I_{N}}(i, j) * K_{l}\right)
$$

$$
Z_{H L}(i, j)=\left(H L_{I_{N}}(i, j) * K_{l}\right)
$$

$$
Z_{H H}(i, j)=\left(H H_{I_{N}}(i, j) * K_{l}\right)
$$

where $1=12,3,4$ and $K_{1}=5 \times 5$ laplacian filter $(1=1,2,3,4)$.

These are tabulated below:

TABLE I: LAPLACIAN FILTERS, $\mathrm{K}_{\mathrm{L}}=(1,2,3,4)$ 
The resultant filtered image is minimum of above four filtered images.

$$
\begin{array}{lll}
R(i, j)=\min \left\{Z_{L H, l}(i, j)\right. & \forall & l=1,2,3,4\} \\
R(i, j)=\min \left\{Z_{H L, l}(i, j)\right. & \forall & l=1,2,3,4\} \\
R(i, j)=\min \left\{Z_{H H, l}(i, j)\right. & \forall & l=1,2,3,4\}
\end{array}
$$

Step4: In thresholding process the above filtered image $\mathrm{R}$ values are compared with the below process:

$$
\begin{aligned}
& D_{L H}(i, j)=\left\{\begin{array}{cll}
\text { median }\left(L H_{I_{N}}(i, j)\right) & \text { if } & R(i, j)=0 \text { or } 255 \\
\operatorname{LH}_{I_{N}}^{3 \times 3}(i, j) & \text { otherwise }
\end{array}\right. \\
& D_{H L}(i, j)=\left\{\begin{array}{cll}
\operatorname{median}\left(H L_{I_{N}}(i, j)\right) & \text { if } & R(i, j)=0 \text { or } 255 \\
H L_{I_{N}}^{3 \times 3}(i, j) & \text { otherwise }
\end{array}\right. \\
& D_{H H}(i, j)=\left\{\begin{array}{cll}
\operatorname{median}\left(H H_{I_{N}}(i, j)\right) & \text { if } & R(i, j)=0 \text { or } 255 \\
H_{I_{N}}^{3 \times 3}(i, j) & \text { otherwise }
\end{array}\right.
\end{aligned}
$$

The resultant denoised image ' $\mathrm{D}$ ' is obtained with better quality.

Step5: Apply IDWT on LL and processed high transformed sub bands: $D_{L H}, D_{H L}$, and $D_{H H}$

$$
D \in\left(L L_{I_{N}}, D_{L H}, D_{H L}, D_{H H}\right)
$$

STEP 6: Finally the denoise image D is extracted from the above equation.

Note: In the case of color image: The RGB components are individually processed with proposed denoising also. The resultant components are concatenated to acquire denoised color image.

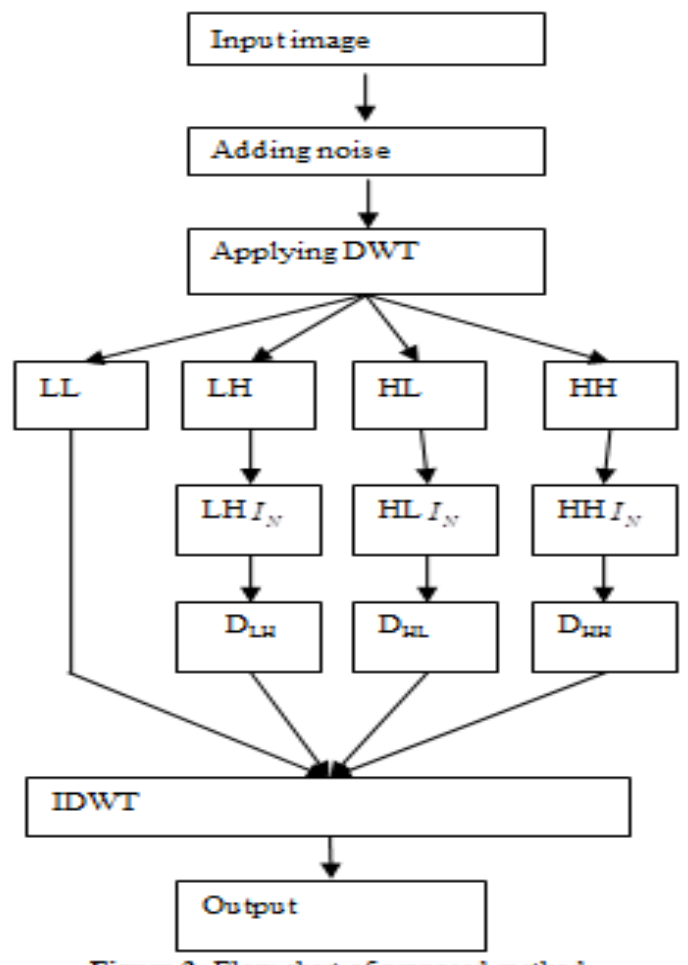

Figure 3, Elows chart of proposed method.
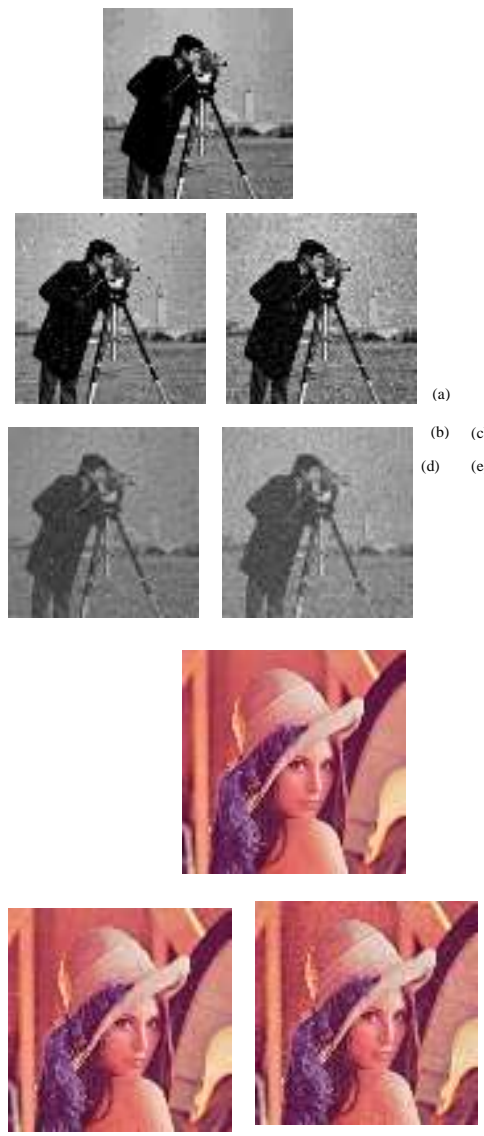

(a)

(b) $\quad(\mathrm{c})$

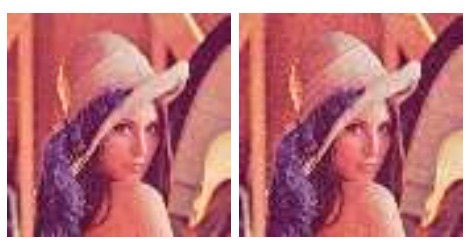

(d) (e)
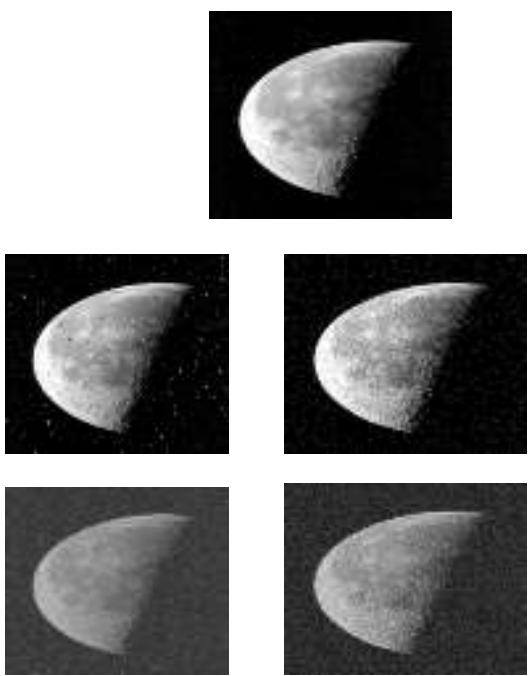

(a) $\mid$
(b) $\mid$ (c)
d) $\mid$ (e)

Fig. 4:. (a) Original image, (b) salt \& pepper noise image, (c) salt \& pepper Denoised image, (d) Gaussian noise image, (e) Gaussian Denoised image.

\section{Experimental Results}

The proposed method was tested with different images [cameraman $(256 \times 256)$,rice $(256 \times 256)$, coins $(246 \times 300)$,pepp ers $(384 \times 512 \times 3)$, moon $(537 \times 358), p_{\text {out }}(291 \times 240)$,pears $(486 \times 732 \times 3)$ and lena ( on a PC with windows7 ultimate, an Intel (R) 
Core(TM)3 CPU , 4GB of RAM and hard disk of 500GB.The software used for this paper is MATLAB and the version 7.8.0.347 (R2009a) with 32 bit (win 32). The resultant denoised images of proposed method are show in figure.4.

For all images the proposed method is verified with various noise densities of salt \& pepper and Gaussian. The results are compared with reference [1]. This is also one of my papers work addressed in time domain analysis. In this paper the authors are worked in transform domain and the results are compared. It proven that transform domain is giving better results than time domain [1] analysis approach.The resultant figure.4. Shows the effectiveness of the proposed denoised technique visibility.

The quantitative performance of the proposed method is also verified with PSNR (peak signal to noise ratio), MI (mutual information) [13] with various noise densities of salt $\&$ pepper and Gaussian noises and tabulated in Table 2 and Table 3 with densities of $1 \%, 5 \%$ and $10 \%$.

\section{A. Peak signal to Noise Ratio (PSNR)}

PSNR (Peak Signal to Noise Ratio) is difference between denoised image and corrupting noise image.It is used to measure the quality of reconstructed image.

$$
\operatorname{PSNR}(d b)=10 * \log _{10}\left[\frac{(255)^{2}}{\frac{1}{M N} \sum_{m=0}^{M-1} \sum_{n=0}^{N-1}\left(D(i, j)-I_{N}(i, j)\right)}\right]
$$

Where $\mathrm{D}(\mathrm{i}, \mathrm{j})$ is the denoised output and $\mathrm{I}(\mathrm{i}, \mathrm{j})$ is the original input image.

\section{B. Mutual Information (MI)}

Mutual information is one of the performance metric ofan image.Here, MI measures the information that reference and the denoising image share:

$$
M I_{D I}=\sum_{i=1}^{L} \sum_{j=1}^{L} P_{D I}(i, j) \log _{2} \frac{P_{D I}(i, j)}{P_{D}(i) P_{I}(j)}
$$

Where $\mathrm{P}_{\mathrm{DI}}$ is the joint probability of images $\mathrm{D}$ and $\mathrm{I}$, and $\mathrm{P}_{\mathrm{D}}$ and $\mathrm{P}_{\mathrm{I}}$ are the normalized probabilities of the two images.

Table II: Quantitative performance of proposed method of PSNR (Peak Signal to Noise Ratio) with various densities

\begin{tabular}{|l|l|l|l|l|l|l|}
\hline \multirow{3}{*}{ Image } & PSNR & \multicolumn{6}{|l|}{} \\
\cline { 2 - 7 } & S\&P*1 & S\&P & S\&P & $\mathrm{G}^{*}$ & $\mathrm{G}$ & $\mathrm{G}$ \\
& $\%$ & $5 \%$ & $10 \%$ & $1 \%$ & $5 \%$ & $10 \%$ \\
\hline \multirow{2}{*}{ Moon } & 23.232 & 16.646 & 13.629 & 21.558 & 15.162 & 12.501 \\
& 9 & 1 & 8 & 3 & 8 & 3 \\
\hline \multirow{2}{*}{ Pout } & 25.286 & 18.788 & 15.824 & 19.944 & 13.403 & 11.092 \\
& 3 & 0 & 2 & 2 & 2 & 5 \\
\hline \multirow{2}{*}{ Pears } & 24.725 & 18.278 & 15.253 & 19.918 & 13.655 & 11.398 \\
& 0 & 4 & 6 & 9 & 5 & 2 \\
\hline \multirow{2}{*}{ Peppers } & 23.955 & 17.807 & 14.927 & 19.943 & 13.948 & 11.622 \\
& 6 & 4 & 5 & 8 & 9 & 0 \\
\hline \multirow{2}{*}{ Lena } & 23.570 & 17.634 & 14.706 & 19.955 & 14.145 & 11.773 \\
& 9 & 3 & 0 & 4 & 0 & 4 \\
\hline \multirow{2}{*}{ Coins } & 22.620 & 17.543 & 14.976 & 19.213 & 13.764 & 11.541 \\
& 8 & 9 & 8 & 9 & 4 & 6 \\
\hline \multirow{2}{*}{ Rice } & 22.356 & 17.617 & 15.214 & 18.867 & 0.8369 & 11.198 \\
\hline Camerama & 1 & 5 & 2 & 9 & 3 \\
n & 20.741 & 16.973 & 14.427 & 18.400 & 13.444 & 11.302 \\
\hline
\end{tabular}

*S \&P: Salt and Pepper Noise, ${ }^{*}$ G: Gaussian Noise
Table 3: Quantitative performance of proposed method of MI (mutual

\begin{tabular}{|c|c|c|c|c|c|c|}
\hline \multirow[b]{2}{*}{ Image } & \multicolumn{6}{|l|}{ MI } \\
\hline & $\mathrm{S} \& \mathrm{P}^{*} 1 \%$ & $\begin{array}{l}\text { S\&P } \\
5 \%\end{array}$ & $\begin{array}{l}\text { S\&P } \\
10 \%\end{array}$ & $\begin{array}{l}\mathrm{G}^{*} \\
1 \%\end{array}$ & $\begin{array}{l}\mathrm{G} \\
5 \%\end{array}$ & $\begin{array}{l}\mathrm{G} \\
10 \%\end{array}$ \\
\hline Peppers & 2.92 & 2.97 & 2.96 & 1.51 & 0.68 & 0.49 \\
\hline Pears & 2.83 & 2.905 & 2.89 & 2.83 & 0.54 & 2.89 \\
\hline Cameraman & 2.58 & 2.61 & 2.58 & 1.45 & 0.99 & 2.58 \\
\hline Lena & 2.56 & 2.61 & 2.59 & 1.50 & 0.63 & 0.42 \\
\hline Coins & 2.36 & 2.38 & 2.36 & 1.11 & 0.78 & 2.36 \\
\hline Moon & 2.11 & 2.13 & 2.10 & 1.42 & 0.95 & 2.10 \\
\hline Pout & 1.60 & 1.64 & 1.64 & 0.62 & 0.29 & 1.64 \\
\hline Rice & 1.29 & 1.34 & 1.35 & 0.81 & 0.52 & 1.35 \\
\hline
\end{tabular}
information) with various densities

*S \&P: Salt and Pepper Noise, ${ }^{*} \mathrm{G} \quad$ : Gaussian Noise

\section{Conclusion}

The proposed method well discussed about the denoisy filtered technique in transform domain, with the proposed method the Salt $\&$ pepper, Gaussian noises are removed effectively from noisy images. The proposed work is also verified in spatial domain [1]. The algorithm is verified with various density values of $1 \%, 5 \%$, and $10 \%$. The performance of the proposed method is observed with PSNR and MI values and compared with existing method. This proven the efficiency of the proposed work in quantitative and qualitative manner. The performance is quite good for images with lower densities of salt \&pepper and Gaussian noises.This can be extendable with the selection of different filtering methods with various transform techniques.

\section{References}

[1] Ch.Hima Bindu, K.Sumathi, "Denoising of Images With Filtering and Thresholding " International conference on research in Engineering Computers \& Technology, PP:142-146, ISBN:597881-908388-7-0, sep, 8-10, 2016, Thiruchy, Tamilnadu, INDIA.

[2] Jyotsna Pati, et al., "A Comparative Study of Image Denoising Techniques", IJIR S, Engineering and Technology, Vol. 2, Issue 3 , March 2013, ISSN: 2319-875.

[3] Noble, et al., "A Comprehensive Review of Image Enhancement Techniques", journal of computing, volume 2, issue 3, march 2010 , issn 2151-9617Gonzanez.c,Richard..E,"Digital Imahe Processing”, 3rd edition,pearson.

[4] K.Jain, "Fundamentals of digital image processing". PrenticeHall, 1989.

[5] Mukesh C.et al., "Survey of Image Denoising Techniques".

[6] R. Yang, et al., "Optimal weighted median filters under structural constraints," IEEE Trans. Signal Processing, vol. 43, pp. 591-604, Mar 1995.

[7] Ben Hamza, et al., "Removing noise and preserving details with relaxed median filters," J. Math. Imag. Vision, vol. 11, no. 2, pp. 161-177, Oct. 1999.

[8] A.K.Jain,Fundamentals of digital image processing. PrenticeHall,1989.

[9] David L.Donoho et al., "Ideal spatial adaption via wavelet shrinkage", Biometrika, vol.81, pp 425-455, September 1994.

[10] David L. Donoho et al., "Adapting to unknown smoothness via wavelet shrinkage", JASA, vol.90, no432, pp.1200-1224, December1995. National Laboratory, July 27, 2001

[11] S.Gopi krishna, et al., " Removal of High Density Salt and Pepper Noise Through Modified Decision Based Unsymmetric Trimmed Median Filter" ISSN: 2248-9622, Vol. 2, Issue 1, Jan-Feb 2012, pp.090-094.

[12] J. Romberg, et al.,"Bayesian wavelet domain image modeling using hidden Markov models," IEEE Transactions on ImageProcessing, vol. 10, pp. 1056-1068, July 2001.

[13] Ch.Hima Bindu et al., "Performance Analysis of Multi Source Fused Medical Images Using Multiresolution transforms", (IJACSA), Vol. 3, No. 10, 2012. 